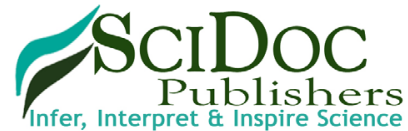

International Journal of Veterinary Health Science \& Research (IJVHSR)

ISSN 2332-2748

\title{
Cryptosporidiosis: Its Importance and Diagnostic Techniques in Farm Animals
}

Review Article

Kerie $\mathrm{Y}^{*}$

Doctor of Veterinary Medicine (DVM) and Master of Microbiology (MSc), Bahir dar Animal Health Diagnostic and Investigation Laboratory (BAHDIL), Ethiopia.

Abstract

It is not strange to see animals suffering from diarrhea. But what is really strange and challenge is identifying the exact cause of that diarrhea with appropriate diagnostic methods and attaching the patient with the corresponding treatment. Wide varieties of etiologies are incriminated as a cause of diarrhea in different animals. Consequently, to identify which causative agent causes this diarrhea, special type of diagnostic methods are required. Veterinarians must give special attention for the cryptosporidial infection since cryptosporidial oocysts are directly infective when shed in the faeces and also caution must be given to avoid accidental infection seeing as it is a zoonotic disease. Cryptosporidiosis is an intracellular protozoal disease that cause diarrhea in neonatal animals. But can infect adult animals which cannot exhibit sings of disease except immunocompromised patient. They can excrete oocysts that may contaminate the environment. The diagnosis of Cryptosporidium infection are usually started based on faecal flotation with Schearer's solution (saturated sucrose solution) followed by staining for the oocysts with modified Ziehl-Neelsen stains. Cryptosporidium antigen in faeces can also be detected by using enzyme- linked immunosorbent (ELISA) or immunofluorescent (IFA) assays and molecular techniques (PCR) which have been described for detection and typing of cryptosporidial genotypes/subtypes. Phase-contrast microscope and fluorescence microscope are required for auramine stain or with immunofluorescence for more rapid diagnosis of cryptosporidiosis but for developing countries like Ethiopia the more appropriate and recommended examination technique for diagnosis of Cryptosporidium is modified Ziehl-Neelsen staining method because of the cost assessments.

Keywords: Cryptosporidiosis; Diagnosis.

\section{Introduction}

Cryptosporidiosis is an intracellular protozoan disease caused by parasites of the genus Cryptosporidium (family Cryptosporidiidae, order Eucoccidiorida, subclass Coccidiasina, class Sporozoasida and phylum Apicomplexa). Amphibians, reptiles, birds and mammals serve as hosts for species of Cryptosporidium [18]. The genus Cryptosporidium was first described in 1912 in the small intestine of mice, but only in 1971 was it associated with an outbreak of bovine diarrhea [1]. The agent has zoonotic importance and a worldwide distribution which may cause diarrhea in neonatal animals including calves, lambs, kids, foals, piglets and other neonates [14].

Cryptosporidium is classified as an emerging pathogen by Centers for Disease Diagnosis and Prevention. The agent has small size oocysts $(4-6 \mu \mathrm{m})$ which leads to diagnostic challenges [3,
15]. Normally, diagnosis of Cryptosporidium is based primarily on identification of oocysts in the faecal sample numerous techniques, conventional microscopic methods to molecular techniques, are available for detection of Cryptosporidium infection [18]. It is possible that Cryptosporidium infection is under diagnosed if specific testing is not requested although many laboratories now routinely test for Cryptosporidium in all faecal samples of patients with diarrhea rather than restricting tests to specific request or to those with recognized risk factors. Several studies have shown that Cryptosporidium infection is not truly rare when looked for with appropriate assays. Microscope of stained faecal smears using auramine phenol or modified acid-fast stain is most widely used in United Kingdom's [19]. But in developing countries the more appropriate and recommended examination technique for diagnosis of Cryptosporidium is modified Ziehl-Neelsen staining method as studied by Venu [18] in India. In study by De Graafa [4] the economic assessment of coprological diagnostic methods

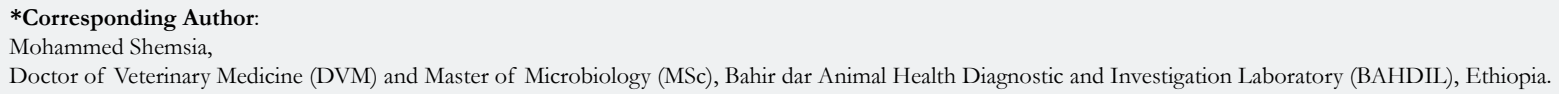

Copyright: Kerie $\mathbf{Y}^{\circ}$ 2019. This is an open-access article distributed under the terms of the Creative Commons Attribution License, which permits unrestricted use, distribution and reproduction in any medium, provided the original author and source are credited. 
and PCR for detection of Cryptosporidium species in bovines was evolved and concluded the low cost involvement of conventional techniques over molecular techniques. Therefore, the objectives of this seminar paper are:

To review on cryptosporidiosis and its various diagnostic techniques in farm animals

$>$ To signify veterinary and zoonotic importance of cryptosporidiosis

\section{Literature Review}

\section{Biology}

Taxonomy and nomenclature: Cryptosporidium species, like Isospora, and Toxoplasma species, are members of the phylum Apicomplexa. Cryptosporidium species were initially distinguished by their size, host specificity, and site of infection within the animal. However; recently, molecular techniques have been used to establish a genetic basis for these distinctions [9]. Therefore, the taxonomic status of the genus Cryptosporidium has been aligned with the coccidian and the taxonomic position of this genus is as follows:

Phylum: Apicomplexa

Class: Conoidasida

Subclass: Coccidiasina

Order: Eucoccidiorida

Suborder: Eimeriorina

Family: Cryptosporidiidae

Genus: Cryptosporidium

Species: currently there are thought to be more than 10 valid species of Cryptosporidium: Cryptosporidium parvum (ruminants and humans), $C$. canis (dog), C. felis (cat), C. andersoni (cattle), C. meleagaridis(birds and bumans), C. galli(birds), C. wrari(guinea pigs), C. hominis (buman), C. baiyleyi (chicken and other birds), C. molnari (fish), C. muris (rodents) [16, 17, 21].

Cryptosporidium is a ubiquitous coccidian organism and constitutes many different species of which C.parvum is widespread in mammals including humans; in calves and lambs. However, genetic methods can be used to differentiate different isolates of C. parvum. So speciation of Cryptosporidium is difficult unless using different diagnostic techniques such as; molecular and immunological techniques that include the use of immunofluorescence or ELISA, and more recently DNA based techniques has been used for the molecular characterization of Cryptosporidium species [16].

Morphology: Mature oocysts are ovoidal or spheroidal;

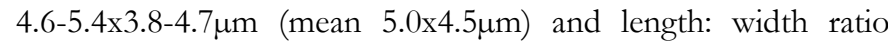
of 1:19 may observe after the demonstration of Ziehl-Neelsen stained faecal smears in which the sporozoites appear as bright red granules [16].

Life cycle: The life cycle of Cryptosporidium differs from most other coccidian organisms. Cryptosporidium species complete their life cycle in a single host with stages of development (sexual and asexual) similar to those of the coccidian genera Eimeria and Isospora. Oocysts containing four infectious sporozoites are excreted in faeces. Oocysts of some species such as Cryptosporidium parvum can remain infectious in cool, wet conditions for 6 months or longer [6]. After ingestion with contaminated water or food from fomites or from direct contact with infected animals, oocysts excyst and sporozoites are released in the small intestine. The released infective sporozoites then infect epithelial cells and become enclosed as trophozoites within parasitophorous vacuole of the microvillous surface of enterocytes rather than in the cytoplasm. Here, all subsequent endogenous stages are intracellular but extracytoplasmic, appearing to rest on the surface of villar epithelial cells $[6,20]$. The trophozoites proliferate (asexually) by merogony to produce, sequentially, two types of meronts within 24 hrs: type I meronts (containing eight merozoites) leave the parasitopharous vacuole to invade other epithelial cells where they develop into more type I meronts or type II meronts (containing four merozoites). The type II meronts do not undergo merogony but produce sexual reproductive stages (male or female gamonts). The zygote formed by sexual reproduction or fertilization (gametogony between male microgamonts and female macrogamonts) from either thick-walled (excreted in the faeces) or thin-walled (autogenous reinfection) oocysts each containing four sporozoites (Greene, 2006). The thick walled oocysts are infective immediately on passing from the host [7]. The oocysts of Cryptosporidium spp can sporulate within the host

Figure 1. Lifecycle of Cryptosporidium spp.

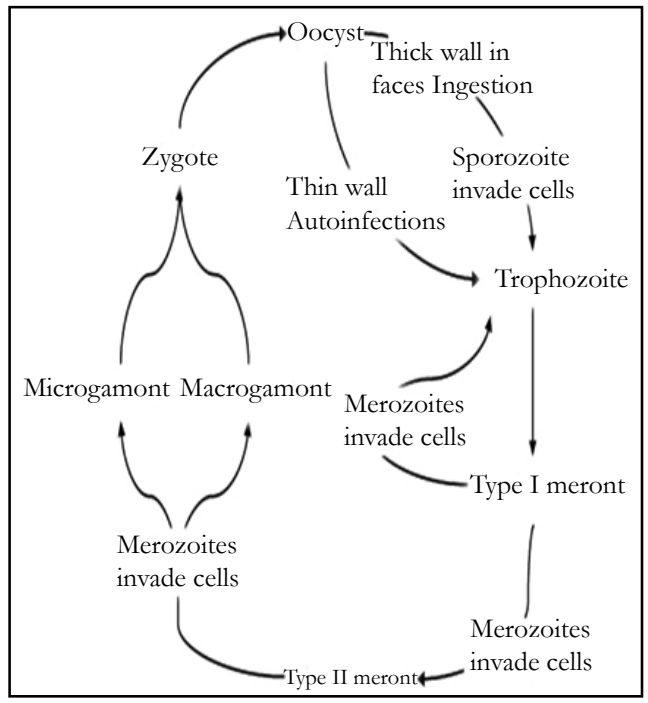


cell, in contrast to the oocyst of Eimeria and Isospora species which do not sporulate until they are infective when passed in the faeces [14].

\section{Transmission and Epidemiological Factors}

Oocysts of cryptosporidia are highly resistant and the transmission occurs via the faecal-oral route. The best-documented routes of transmission are waterborne, food borne, and animal-to-animal spread. Faecal contamination of food or drinking water is a common source of infection and large outbreaks can occur when a population/community water source becomes contaminated (Greene, 2006). The source of infection is faeces which contains oocysts that are fully sporulated and infective when excreted. Large numbers of oocysts are excreted during the patent periods in calves resulting in heavy environmental contamination. In farm animals, transmission may occur directly from animal to animal, indirectly via fomites, from contamination in the environment or faecal contamination of the feed or water supply [15].

Infection into the environment of newborn animals and an increase in contamination of their immediate environment occur as the result of a peri-parturient rise in the faecal excretion of oocysts by the dam. This has been recorded in ewes, in beef cattle herd and in some dairy herds [4, 14]. The oocysts of cryptosporidia shed in faeces in highly resistant to environmental stress and, because it is excreted from the host fully sporulated; it is capable of direct transmission by the faecal-oral rout rapidly from lambs to lambs or ewes to lamb, particularly in communal housing and overcrowded or via contaminated water or from mother to young via the udder when they are contaminated with infected young animal faeces in the lying area of the dams [4]. This might explain the association between cryptosporidial diarrhea and the farm type and/or its specific hygienic conditions. Within 5-10 days of becoming infected, a single lamb can spread infection to a large proportion of the lamb crop. Cryptosporidium infections are more common in rainy season, large herds and in farms with multiple female animal maternity facilities [4]. Rodents can be potential reservoir of infection for livestock enterprise and mechanical transmission via birds, insects and man may also be involved [1]. Cryptosporidial oocysts are also resistance to drying and may persist for at least 45 days in animal faeces [16]. Infection occurs by ingestion of thick walled oocysts; these may survive in the environment for months and the sporulated oocysts containing four infectious sporozoites is excreted in faeces and is the infectious agent for a susceptible host [11].

\section{Cryptosporidium infection and Cryptosporidiosis}

Cryptosporidium is an intracellular protozoal parasite that associated with gastrointestinal disease and inhabits the epithelium of the respiratory and digestive system of different animals. Infections of the ileum are most common but gastric, respiratory and conjunctiva infections have been observed in immunosuppressed hosts. Most species may be relatively host specific, for example Cryptosporidium found in reptiles and birds apparently do not infect mammals [8]. C. parvum primarily infects the small intestine of mammals but in lambs, it also sometimes infects the caecum, colon and rectum [1].

Cryptosporidiosis may cause malabsorption or secretory diarrhea, but the underling mechanisms are complex and still not fully understood and have been reviewed elsewhere. The organism remains just beneath the luminal cell membrane of the intestinal epithelial mucosa. Functional impairment (glucose-stimulated sodium and water absorption) and morphological changes (villous atrophy, crypt hyperplasia, and inflammatory cell infiltration) have been reported in pigs experimentally infected with C. parvum [8]. The parasite commonly co-exists with other enteropathogens, especially rotavirus and corona virus, to produce intestinal injury and diarrhea, and epidemiological studies suggest that diarrhea is more sever in mixed infection [6, 9, 20]. Generally, case fatality rates in cryptosporidiosis are low unless complicated by other factors (for example, concurrent infection, energy deficits from inadequate intake of colostrums and milk, chilling from adverse weather conditions) [10]. The pathogenesis of diarrhea is unknown, but the varying degrees of villous atrophy suggest that digestion and absorption may be impaired, resulting in diarrhea [14].

\section{Clinical and Pathological Features}

Varying degrees of diarrhea are common features in all species of animals including human beings affecting by Cryptosporidium. Calves usually have a mild to moderate diarrhea that persists for several days regardless of treatment [10] and can show depression, dehydration, anorexia and tenesmus. Variable levels of morbidity have been reported and mortality is generally low except concurrent infection [15]. In neonatal lambs (4-9 days old) causes a profuse watery diarrhea which, in welled-fed animals, often persist for 5-7 days before lessening and the lambs then recover. The scouring often leads to dehydration, in appetence, abdominal tension and lethargy. Diarrhea frequently coincides with the onset of oocysts shedding 3-7 days after ingestion of oocysts and peak out puts can range from 105 to 107 oocysts per gram of faeces. In sever case, lambs may die within 2-3 days of the onset of diarrhea but adult sheep can undergo non-clinical infection, shedding low number of oocysts, particularly around parturition, thus acting as a reservoir of infection for the susceptible lamb crop [1]. Generally, affected lambs and kids are often active, alert and nursing. The diarrhea is usually very liquid and yellow which can vary from mild and self-limiting to sever, especially with mixed infection. Relapse is quite common in calves, lambs and kids [13]. In poultry, the parasite is fairly common, infecting digestive, respiratory tracts and the bursa of fabricius and is a primary pathogen [8]. In animals, upon necropsy, villi are shorter than normal and there also crypt hyperplasia. Histologically, large numbers of the parasite are embedded in the microvillus of the absorptive enterocytes [14].

Generally, the typical clinical manifestation in immunocompetent host is watery diarrhea and, or mucoid diarrhea with or without abdominal pain lasting up to 4 weeks. Cryptosporidium infection cause sporadic case of water-related outbreaks consisting of self-limited diarrhea in immunocompetent hosts. In contrast, it may cause chronic and even life-threatening illness in immunocompromised patients [19].

\section{Economic Significances}

The economic losses due to cryptosporidial infections of neonatal calves are related to diarrhoea: dehydratation, growth retardation and to a lesser extent mortality [14]. Diarrhoeic problems of calves demand special care: feeding of electrolyte solutions, i.e. 
fluid therapy, drug administration, hygienic measures, etc. which are costly as well as labour and time-consuming. For instance, in Belgium, mortality due to the neonatal diarrhoea syndrome is estimated between $5 \pm 10$ percent. Cryptosporidium parvum is considered as the most commonly found enteropathogens in calves during their first weeks of life. The parasite frequently acts alone, but the losses are more severe when concurrent infections occur as has been demonstrated for viral and bacterial enteropathogens. In the absence of other enteropathogens, mortality is higher in lambs than in calves and morbidity can reach 100\% [4].

\section{Zoonotic Potential and Public Health Significance}

Cryptosporidial infections in people were not recognized until 1976 [8] which are considered to be relatively common non-viral cause of self-limiting diarrhea in immunocompetent persons, particularly children. In immunocompromised person, clinical disease may sever. This is particularly true in case of human patient with HIV/ADIS [10]. Individuals with congenital immunodeficiency disease may survive for years with cryptosporidiosis when fed parenterally. Immunocompetent persons may be infected readily with Cryptosporidium as when more people in developing countries developed diarrhea as the result of constructing the organism through the public water supply. Infection among veterinary students is common after contact with infected calves, lambs, dogs and cats [8]. So that animal handlers on a calf farm can be at high risk of diarrhea due to cryptosporidiosis transmitted from infected calves and immunocompromised people should be restricted from access to young animals and possibly from access to farms [14].

\section{Treatment, Prevention and Control}

Treatment: Infections in young and immunocompromised animals as well as peoples are usually self-limiting, and full recovery soon ensues. To date, few drugs have been used successfully for treating cryptosporidiosis, although more than 100 drugs have been screened. The amino glycoside antibiotics, paromomycin is effective in treating acute intestinal case of cryptosporidiosis in people, calves, mice and rats $(125-165 \mathrm{mg} / \mathrm{kg}$ po of $12 \mathrm{hr}$ for 5-8 days). Paromomycin was no more effective than placebo in treating human patients with advanced AIDS [8]. Halofuginone lactate is licensed for therapeutic and prophylactic treatment of cryptosporidiosis in calves at a dose rate of $1 \mathrm{mg} / 10 \mathrm{~kg}$, but not for use in small ruminants. Treatment may reduce clinical signs and diminishes oocyst outputs, but does not eradicate infection. Symptomatic treatment may be given in the form of antidiarrheal and fluid replacement therapy $[1,16]$. Therapy with halofuginone significantly reduced the severity of diarrhea and dehydration compared with sulfadimidine therapy in calves. Halofuginone prevents reinfection of the gut by sporozoites and recycling merozoites. Paromomycin sulfate give orally at a dose of $100 \mathrm{mg} /$ $\mathrm{kg}$ Bw daily for 11 consecutive days from the second day of age proved successful in preventing natural disease in a controlled clinical field trail in goat kids and to reduce, but not completely prevent diarrhea in infected lambs [14].

Prevention and Control: The importance of colostrum in protecting neonatal ruminants against cryptosporidium infection is a very valuable point to address the problems. On the other hand from a perspective of disease control, preventive hygiene measures are the most important tools in the struggle against cryptosporidiosis in farm animals, the objective being to destroy external forms of the parasite and to prevent their transmission among animals and from the environment to the host [10, 14]. In ruminant husbandry, the destruction of oocysts in the pens and buildings used for parturition by applying moist heat and/ or chemical disinfectants, the use of abundant clean straw beds, avoidance of high stocking rates in the parturition area and the separation of healthy and ill animals during out breaks of diarrhea, in addition to the administration of appropriated supplies of colostrum to neonates, all help to prevent outbreaks of cryptosporidiosis and to minimize mortality and morbidity in infected herds [4].

\section{Diagnostic Approaches of Cryptosporidiosis in Farm Animals}

\section{General Aspects}

Because cryptosporidial oocysts are directly infective when shed in the faeces, caution must be used to avoid accidental infection. Samples should be placed in a non-breakable container and the outside of the container should be disinfected to avoid accidental infection of laboratory personnel's. The laboratory should be notified of suspicion of cryptosporidial infection oocysts may be seen microscopically on direct smear of faeces (8), but Cryptosporidium spp. are much smaller than Eimeria spp. and are therefore difficult to detect in faecal flotation. Laboratory techniques that use acid-fast stains or immunological techniques greatly aid detection. Due to small oocysts (4 to $6 \mu \mathrm{m}$ in diameter), they are easily missed on a faecal smear. If the faecal smears do not concentrate the oocysts, this technique is less sensitive than faecal flotation [15]. So that concentration techniques, such that Sheather's sugar flotation or formaldehyde-ether sedimentation, or by staining faeces with Kinyoumcarbolfuchsion stain or modified acid fast stain [2] and immunoassays have been developed for the detection of cryptosporidia.

The faecal concentration methods are often necessary to reveal the presence of oocysts with these techniques. Sugar or salt solutions are used [8]. In Africa, the standard approach to diagnosis is faecal microscopy. The smear are prepared and stained with modified Ziehl-Neelsen (modified acid fast) stain (which is similar to the detection of acid fast bacteria but without warming the slide) oocysts stain red on a blue background. The technique is slow and requires skill as the oocysts must be differentiated from yeast and other spores with which may cause confusion. So more rapid diagnosis may be achieved with an auramine stain or with immunofluorescence, but both of this requires a fluorescence microscope and the diagnosis should still be confirmed with the ZN stain. Other tests, such as antigen detection tests or PCR expensive and not yet proven to be of value; in Lusaka they have found these tests to be insensitive [12]. But PCR is highly sensitive and has the advantage of showing which Cryptosporidium genotype is involved and it is useful in epidemiological investigation [19].

\section{Types of Diagnostic Techniques}

The diagnosis of Cryptosporidium infection in farm animals is usually based on faecal flotation with Schearer's solution (sucrose) followed by staining for the oocysts with Modified ZiehlNeelsen stain [15]. Cryptosporidium antigen in faeces can be also 
detected by using enzyme - linked immunosorbent (ELISA) or immunofluorescent (IFA) assays (Aitken, 2007) and molecular techniques (PCR) which have been described for detection and typing of cryptosporidia [15]. The oocysts are small $(5-6 \mu \mathrm{m}$ in diameter) and difficult to detect by normal light microscope, rather detected by phase-contrast microscope [14].

\section{Microscopic based techniques:}

Floatation: The flotation principle uses a liquid suspending medium (sucrose solution), which is denser than the oocysts to be concentrated. Therefore, when mixed with flotation fluid, the cryptosporidium oocysts rise to the surface and can be skimmed out of the surface film and detected under high power microscope [10]. For a flotation fluid to be useful in diagnostics, when morphology and morphometry are the critical factors, the suspending medium must not only be heavier than the oocysts to be floated but must not produce shrinkage sufficient to render the oocysts undiagnosable [16]. Generally these methods are suitable for concentrating cryptosporidium oocysts. Since the solution have high specific gravity than the cryptosporidium oocysts [15].

Modified Ziehl-Neelsen staining: Cryptosporidium oocysts will appear as pink stained, round to oval structures of about 3 to $6 \mu \mathrm{m}$ in diameter, containing distinct internal structures while the samples are staining with modified Ziehl-Neelsen staining. The modified Ziehl-Neelsen staining is a time-consuming method (about 30 to 45 minutes), which requires intensive training and experience to interpret the results but it is less expensive as compared to other techniques [5]. It needs Care to be used in interpretation, particularly of preparations stained with modified Ziehl-Neelsen staining, as a variety of structures can be confused with oocysts (so-called cryptosporidium-like bodies). These include fungal spores which are generally larger (6-10gm) than oocysts (4-6 m), including mould spores (such as some Mucorspecies), and the spores of the common mushroom: fat globules and bacterial spores have also been mistaken for oocysts by some workers, although these can be distinguished clearly by size alone. But it is widely used than other techniques due to its low cost technique [3].

\section{Immunological based detection methods:}

Enzyme Linked Immunosorbent Assay (ELISA): Antigen based ELISA have several significant advantages over other methods currently used for diagnosis of cryptosporidium oocysts [5] including excellent sensitivity and specifity, use of 96 well plate formats which enhance their potential as large scale screening tools in epidemiological studies such as water born outbreak situations, widely used and simple to perform with minimal labor. However, the expensive nature the enzyme immunoassay kits used for ELISA place them above the reach of many laboratories especially in developing country like Ethiopia [5].

Immunofluorescent Assay (IFA): This technique offers the highest combination of sensitivity and specificity to estimate the Cryptosporidium oocysts in farm animals and the detection and identification of Cryptosporidium oocysts can be achieved using monoclonal antibody immunofluorescence (IFAT) technique (1) which requires special equipment (fluorescence microscope) and commercially available test kits. However, important challenges facing the usefulness of this method that includes time consuming, allows only few samples to be examined per day and the necessity for an expert or experienced personnel which may not be available to interpret the results [3].

\section{Polymerase Chain Reaction (PCR) based methods:}

Polymerase Chain Reaction (PCR): PCR is sensitive and has the potential for accurate diagnosis in farm animals which do not presently know the reason for their diarrhea. This could have considerable advantage in the treatment of immunocomppromissed farm animals, allowing for easy diagnosis before the onset of clinical signs. PCR technique is rapid and accurate and the obtained results are also easy to interpret [16]. Furthermore PCR test is capable of directly differentiating between different farm animal derived genotypes of Cryptosporidium on the basis of the PCR product [20]. PCR technique cannot however, be used for routine diagnosis of Cryptosporidium oocysts due to the following challenge including its technical complexity and interference of results by inhibitors (like bilirubin, bile salt and complex polysaccharides) which reduce its sensitivity but these inhibitors can be eliminated by Cryptosporidium oocysts purification from fecal sample which involves density gradient concentration of fecal samples [16].

\section{Conclusion and Recommendation}

Cryptosporidium infection is one of the most important causes of diarrhea in different newborn animals, even in children which is zoonotic. The major way of transmission for this agent is fecal-oral route through contaminated food and water by fecal matter that contain the oocyst of cryptosporidium because the agent is very resistant to environmental change and to different disinfectant. Faeces are the most crucial sample for the diagnosis of these diseases while during collection special attention will require. After submitting the sample to the laboratory different diagnostic methods are used for the diagnosis of cryptosporidiosis. Of which, faecal immunoassay are the preferred method for diagnosis of cryptosporidium for pet animal sample but in ruminants especially for bovines faecal flotation with Sheather's solution (sucrose) followed by modified Ziehl-Neelsen stains are applicable. Molecular techniques (PCR) also vital for detection and typing of cryptosporidium which is not widely available in developing country like Ethiopia. Therefore; based on the above conclusion the following recommendations are forwarded:

Faecal samples are required for the diagnosis of Cryptosporidium, therefore during sample collection special caution must be used to avoid accidental infection, because cryptosporidial oocysts are directly infective when shed in the feces, and the sample should be placed in a non-breakable container and the outside of the container should be disinfected to avoid accidental infection of laboratory personnel's.

In developing countries like Ethiopia the more appropriate and recommended examination technique for diagnosis of Cryptosporidium is modified Ziehl-Neelsen staining method due to the low cost involvement of this method.

Veterinarians should give an attention during the detection of Cryptosporidium by using different diagnostic methods, since it is difficult to diagnose due to small size oocysts which may 
lead to false negative results.

Because of water is a prominent vehicle for the transmission of Cryptosporidial oocysts and because of different farm animals graze in the drainage basin source of this water. So that to minimize the contamination of water and risk of such animals, the owners should dispose faecal matter of the animals properly and restrict free movement of farm animals respectively.

Fluorescence microscope and phase-contrast microscope are required to observe stains prepared from faecal samples like auramine and Modified Ziehl-Neelsen stains. But they are very low in number in different laboratories. So that our government should give attention in order to fulfill such diagnostic laboratory facilities.

\section{References}

[1]. Aitken ID. Disease of sheep. 4th ed. Blackwell Publishing. Ames, Iowa. 2007; 179-181.

[2]. Birchard SJ, Sherding GR. Saunders Manual of small Animal Practice. 3rd ed. Saunders of Elsevier, USA. 2006; 717-718.

[3]. Chen XM, Keithly JS, Paya CV, LaRusso NF. Cryptosporidiosis. New England Journal of Medicine. 2002 May 30;346(22):1723-31.

[4]. de Graaf DC, Vanopdenbosch E, Ortega-Mora LM, Abbassi H, Peeters JE. A review of the importance of cryptosporidiosis in farm animals. Int J Parasitol. 1999 Aug;29(8):1269-87. PubMed PMID: 10576578.

[5]. Ayana D, Tilahun G, Wossene A. Study on Eimeria and Cryptosporidium infections in sheep and goats at Elfora export abattoir, Debre-zeit, Ethiopia. Turkish Journal of Veterinary and Animal Sciences. 2009 Nov 18;33(5):36771.

[6]. Fayer R. Xiao L. Cryptosporidium and Cryptosporidiosis. 2nd ed. CRC
Press and IWA Publishing. Boca Raton, FL. 2008; 1-42.

[7]. Fowler ME, Miller RE. Zoo and wild animal medicine: current therapy. Elsevier Health Sciences; 2008. 154-156.

[8]. Greene CE. Infectious diseases of the dog and cat. WB SaundersIElsevier Science. 2006; 785-791.

[9]. Leav BA, Mackay M, Ward HD. Cryptosporidium Species: New Insights and Old Challenges. 2003; 36(7):903-8.

[10]. Kahn CM, Scott L, Aiello SE. The Merck veterinary manual 9th ed. Copyright (C) by Merck Co., Inc printed in the USA by National publishing. Inc. Philadelphia, Pensylvania. 2005:146-8.

[11]. Krauss H, Weber A, Appel M, Enders B, Isenberg HD, Schiefer HG, Slenczka W, von Graevenitz A, Zahner H. Zoonoses: infectious diseases transmissible from animals to humans. Washington, DC: ASM press. $2003 \mathrm{Jul}$; 278-279.

[12]. Parry E, Gadfrey R, Mobey D and Gill G. Principles of Medicine in Africa. 3rd ed. Singapore: Cambridge university press. 2004; 498-499.

[13]. Pugh DG. Sheep and Goat Medicine. 1st ed. USA: Saunders. $2002 ; 82-83$.

[14]. Blood DC, Radostits OM, Gay CC, Hinchcliff KW, Constable PD. Veterinary Medicine: A textbook of the diseases of cattle, horses, sheep, pigs and goats. Saunders Ltd. 10th ed. 2007; 1512-1515.

[15]. Smith BP. Large animal internal medicine. 4th ed. St. Louis: Mosby Elsevier. 2009; 345-352.

[16]. Taylor AM, Coop RL and Wall LR. Veterinary Parasitology.3rd ed. Blackwell SciencePublishing. 2007; 73-74.

[17]. Thompson RCA, Armson A and Ryan UM. Parasitology Laboratory: Basic biology of Cryptosporidium. Elsevier science B.V. Kansas state university. 2008.

[18]. R Venu, BR Latha, S Abdul Basith, C Sreekumar, G Dhinakar Raj, M Raman. Factors influencing on prevalence of Cryptosporidium infection in south Indian dairy calves. J Parasit Dis. 2013 Oct; 37(2): 168-172.

[19]. Woywodt A, Vythelingum K and Cheesbrough J. Clinical Kidney Journal. Oxford. 2012; 5(3):265-8

[20]. Xiao L. and Fayer R. Molecular characterization of species and genotypes of Cryptosporidium and Giardia and assessment of zoonotic transmission. Int J Parasitol. 2008 Sep;38(11):1239-55.PubMed PMID: 18479685.

[21]. Xiao L, Fayer R, Ryan U, Upton SJ. Cryptosporidium taxonomy: recent advances and implications for public health. Clin Microbiol Rev. 2004 Jan;17(1):72-97. PubMed PMID: 14726456. 